\title{
Nova espécie e novos registros para o Brasil de hifomicetos em folhedo submerso coletados no Parque Municipal Alfredo Volpi, São Paulo, SP, Brasil ${ }^{1}$
}

\author{
Carolina Gasch Moreira ${ }^{2}$ e Iracema Helena Schoenlein-Crusius ${ }^{2,3}$
}

Recebido: 23.03.2011; aceito: 26.09.2012

\begin{abstract}
New species and new records for Brazil of Hyphomycetes on submerged leaf litter collected in Parque Municipal Alfredo Volpi, São Paulo, São Paulo State, Brazil). During a study about fungal succession in submerged leaf litter in Parque Municipal Alfredo Volpi, São Paulo, SP, a new species of Pyramidospora and two new records of Triscelophorus acuminatus Nawawi and Blodgetia indica Subram. were found. They were described and illustrated for the first time in Brazil. Key words: reservoirs, taxonomy, urban waters
\end{abstract}

RESUMO - (Nova espécie e novos registros para o Brasil de hifomicetos em folhedo submerso coletados no Parque Municipal Alfredo Volpi, São Paulo, SP, Brasil). Durante o estudo da sucessão fúngica em folhas submersas no Parque Municipal Alfredo Volpi, São Paulo, SP, uma nova espécie de Pyramidospora e dois novos registros de Triscelophorus acuminatus Nawawi e Blodgetia indica Subram. foram encontrados, e são descritos e ilustrados pela primeira vez para o Brasil.

Palavras-chave: águas urbanas, reservatórios, taxonomia

\section{Introdução}

Independentemente da validade taxonômica atual, segundo a qual Deuteromycota, Deuteromycetes, Deuteromycotina ou fungos imperfeitos deixaram de ser considerados como grupo taxonômico (Alexopoulos et al. 1996), os termos "hifomicetos aquáticos" ou "Hyphomycetes aquáticos" ainda são utilizados para designar fungos conidiais que se reproduzem assexuadamente exclusivamente na água. Na literatura encontram-se também os termos fungos tetrarradiados ou fungos ingoldianos, sendo estes últimos assim denominados em homenagem ao Dr. C.T. Ingold, pioneiro no estudo dos mesmos. Trata-se de um grupo artificial de fungos, cujas formas hidrodinâmicas dos conídios, que podem ser estrelados, sigmóides ou esféricos, proporcionam leveza e agilidade na dispersão, principalmente nas águas lóticas, favorecendo a atuação dos mesmos como um dos mais relevantes decompositores de substratos foliares submersos (Ingold 1975, Gulis et al. 2005).

Revisões sobre o estado da arte dos estudos sobre hifomicetos aquáticos nos trópicos (Schoenlein-Crusius
\& Grandi 2003, Schoenlein-Crusius \& Malosso 2007, Schoenlein-Crusius et al. 2009), nos quais os principais estudos taxonômicos e ecológicos referentes a esses fungos foram sintetizados, revelam que a contribuição dos estudos brasileiros para o conhecimento da micota aquática da América do Sul tem sido bastante expressiva. Estima-se que mais de $60 \%$ das citações de hifomicetos aquáticos no continente sul-americano foram efetuadas no Brasil, através do estudo desses fungos associados a diversos tipos de folhas como Ficus microcarpa L., Quercus robur L.f., Alchornea triplinervia (Spreng.) Müll. Arg., Tibouchina pulchra Cogn. ou amostras de folhedo misto, submersas em riachos, córregos ou em lagos com diferentes graus de eutrofização, localizados na Mata Atlântica (Schoenlein-Crusius et al. 2009) ou no Cerrado (Schoenlein-Crusius 2002) ou ainda em águas urbanas, como na Represa do Guarapiranga e sistema do rio Jacaré-Monjolinho (Malosso 1999) ou no Parque Estadual das Fontes do Ipiranga, (Moreira 2006, Moreira \& Schoenlein-Crusius 2007, Schoenlein-Crusius et al. 2009).

Originalmente a presença dos hifomicetos aquáticos foi fortemente associada a córregos

1. Parte da Tese de Doutorado da primeira Autora

2. Instituto de Botânica, Núcleo de Pesquisas em Micologia, Caixa Postal 68.041, 04045-972 São Paulo, SP, Brasil

3. Autor para correspondência: iracema@crusius.com.br 
ou riachos de águas límpidas, bem aeradas, moderadamente turbulentas e frias, estando os seus conídios dispersos nas espumas ou associados a folhas pós-senescentes em decomposição (Ingold 1975). Posteriormente, no entanto, ocorrências desses fungos de forma diversa e abundante também foram registradas nas condições opostas das consideradas ideais (Au et al. 1992, Schoenlein-Crusius et al. 2009). A atividade decompositora das espécies, crescendo e reproduzindo-se ao longo das margens e nervuras de folhas submersas em lagos e represas com diferentes níveis de eutrofização indica que a ocorrência dos mesmos também pode ser relevante nos sistemas aquáticos lênticos. Portanto, o levantamento dos hifomicetos aquáticos justifica-se tanto em águas preservadas quanto nas águas urbanas, cuja diversidade microbiana ainda é pouco conhecida.

\section{Material e métodos}

O Parque Municipal Alfredo Volpi, localizado no Bairro do Morumbi na Zona Oeste da cidade de São Paulo (233' $16^{\prime \prime}$ S e $\left.46^{\circ} 42^{\prime} 09^{\prime \prime} \mathrm{W}\right)$, abrange uma área de $142.432 \mathrm{~m}^{2}$ com vegetação remanescente da Mata Atlântica de Planalto, guarnecida por duas nascentes de águas perenes e três lagos (SVMA 2006).

O lago utilizado para os experimentos localiza-se próximo a entrada do Parque, sendo abastecido por duas nascentes perenes, uma nascente sazonal e três bocas de lobo, que recebem águas pluviais acrescidas de efluentes orgânicos provindos da rua circular ao Parque (SVMA 2006).

Entre os meses de fevereiro a março de 2008, folhas foram coletadas diretamente da serapilheira existente no entorno de um exemplar de Caesalpinia echinata Lam. (pau-brasil) e de um de Campomanesia phaea (O. Berg.) Landrum (cambuci), acondicionando-se as mesmas separadamente, em caixas de papelão para secarem ao ar livre durante duas semanas.

Em abril de 2008, foram coletadas folhas frescas diretamente das mesmas árvores, no intuito de submergi-las no lago, juntamente com as folhas previamente secas ao ar livre, possibilitando a comparação da sucessão fúngica em situações diferentes. As folhas de cada espécie arbórea, em duas situações, isto é, as frescas e as secas ao ar livre, foram acondicionadas separadamente em quatro caixas de plástico $(47,5 \mathrm{~cm}$ de comprimento, $28,5 \mathrm{~cm}$ de largura e 14,0 cm de altura) com tampa removível, previamente forradas internamente com tela de náilon de malha de $1 \mathrm{~mm}$ de diâmetro. As caixas foram preenchidas totalmente com 1,5 a $2,0 \mathrm{~kg}$ de folhas, evitando-se a compactação excessiva das mesmas para melhor circulação da água, submergindo-se uma ao lado da outra, a aproximadamente $50 \mathrm{~cm}$ das margens do lago (Moreira 2011).

Mensalmente, de maio a setembro de 2008, as caixas foram abertas para coletar-se, de cada uma delas, 300 folhas que foram colocadas em sacos plásticos contendo em torno de $500 \mathrm{ml}$ de água do lago para evitar ressecamento durante o transporte ao laboratório. Ali, cada tipo de amostra foi retirada do saco plástico e lavada individualmente sob água corrente para eliminação de detritos. Posteriormente, a amostra foi dividida em três alíquotas, entre as quais uma destinou-se ao estudo dos hifomicetos aquáticos.

Para o isolamento dos hifomicetos aquáticos foram utilizados 100 folhas de C. echinata e 50 de C. phaea, que foram submetidas à técnica de lavagens sucessivas (Pugh et al. 1972, Grandi \& Gusmão 1998), de acordo com a qual foram colocadas em frascos de vidro contendo $50 \mathrm{ml}$ de água destilada esterilizada, agitadas manualmente por 1 minuto e drenadas, repetindo-se esse procedimento por 30 vezes, no intuito de eliminar propágulos aderidos à superfície foliar. Cerca de 20 folhas, assim tratadas, foram colocadas em placas de Petri contendo água destilada esterilizada (Ingold 1975) e incubadas em temperatura ao redor de $20^{\circ} \mathrm{C}$. A cada dois dias (no total de 30 dias), o desenvolvimento de hifomicetos aquáticos nas folhas foi acompanhado pela observação, sob microscópio óptico, de lâminas montadas com água destilada estéril e fragmentos de cada substrato, à procura de estruturas de valor taxonômico, que foram ilustradas em câmara clara e fotografadas para fins de documentação. Após a confirmação da presença de estruturas de reprodução nas folhas, foram montadas lâminas permanentes com o meio de montagem PVLG - álcool polivinílico acrescido de lactofenol e glicerina (Morton et al. 1993), para serem depositadas no Herbário "Maria Eneyda P. Kauffmann Fidalgo" (SP) do Instituto de Botânica, São Paulo, SP. A identificação das espécies de hifomicetos aquáticos foi conduzida por meio de consulta a chaves de classificação e literatura pertinente (Ingold 1975, Marvanová 1997).

\section{Resultado e Discussão}

Dos 43 táxons obtidos no estudo da sucessão de hifomicetos aquáticos, três constituem novos 
registros para o Brasil, incluindo uma nova espécie para a Ciência.

Blodgettia indica Subram, J. Ind. Bot. Soc. 33: 36, 1954.

Figuras 1-3 e 15-16

Hifas hialinas, septadas, pouco ramificadas. Conidióforos eretos, simples, septados, levemente acastanhados, pouco diferenciados das hifas, irregular e esparsamente ramificados. Conídios alongados, fusiformes, torulosos, levemente castanho claro, individuais, terminais ou laterais, formados a partir da diferenciação do conidióforo; 3-5(6) septos transversais, formação sequencial de 3-4 células globosas, esféricas a oblongas a partir do conidióforo até o ápice; $2^{\mathrm{a}}$ ou $3^{\mathrm{a}}$ célula maior e com coloração castanho mais intensa do que a célula terminal, $20-50 \times 7,5-17,5 \mu \mathrm{m}$. Presença de pequena reminiscência do conidióforo $(8-10 \times 3-4 \mu \mathrm{m})$ na extremidade do conídio, melhor distinguível quando este se encontra livre.

A espécie Blodgettia indica foi originalmente descrita por Subramanian (1954) de folhas em decomposição. Posteriormente, foi encontrada associada à folhedo submerso em um lago em Cuyabeno, Equador (Matsushima 1993), em folhas submersas no rio Neryia (Sridhar \& Kaveriappa 1989), e em espumas e folhas submersas no rio Cauvery, ambos na Índia (Rajashekhar \& Kaveriappa 2003). Aparentemente a espécie foi registrada apenas em estudos realizados em ambientes tropicais e sempre em associação com substratos submersos e espumas.

Material examinado: BRASIL. São PAULO: São Paulo, Parque Municipal Alfredo Volpi, 25-V-2008, C.G. Moreira \& I.H. Schoenlein-Crusius s.n. (SP416392).

Habitat: Folhas de Caesalpinia echinata Lam. e Campomanesia phaea (O. Berg.) Landrum submersas em um lago no Parque Municipal Alfredo Volpi, São Paulo, SP, Brasil.

Pyramidospora robusta Moreira \& Schoenlein-Crusius sp. nov. (MB 801371).

Figuras 5-8 e 9-14

Hyphae hyalinae, ramosae, septatae; conidiophora hyalina, gracilia, simplicia, erecta, transverse septata (1,2-1,7 $\mu \mathrm{m}$ diam.), ex hyphis somaticis paulo distinguibilia, in marginibus foliorum immersa, cellulis conidiogenicis terminalibus munita; conidia terminalia, hyalina, luctea ad castaneiformia, ramosa, septata, axi principali brevi $(12-18 \times 6-8 \mu \mathrm{m})$ in duabus cellulis diviso, 8-13 ramificationibus lateralibus simplicibus munito $(20-32 \times 6-8 \mu \mathrm{m})$, septis constrictis gemmis similibus, accretione irregulari, horizontali, verticali vel curvata; conidia matura et libera, 42-57 $\mu \mathrm{m}$, ex apicibus ramificationum, opposita.

Hifas hialinas, ramificadas, septadas; conidióforos hialinos, delicados, simples, eretos, transversalmente septados (1,2-1,7 $\mu$ m diâm.), pouco diferenciados das hifas somáticas, imersos nas margens das folhas, com células conidiogênicas terminais; conídios terminais, hialinos, amarelados a acastanhados, ramificados, septados, constituídos de um eixo principal curto (12-18 × 6-8 $\mu \mathrm{m})$, dividido em duas células com 8-13 ramificações laterais simples $(20-32 \times 6-8 \mu \mathrm{m})$, com septos constritos de aparência semelhante a gomos, crescimento irregular, horizontal, vertical ou curvo. Conídios maduros e livres medem de $42-57 \mu \mathrm{m}$ do ápice de ramificações posicionadas de forma oposta.

O gênero Pyramidospora foi descrito por Nilsson (1962) a partir de espécimes associados com folhas em decomposição submersas. Originalmente, o autor registrou a espécie tipo do gênero $P$. casuarinae para a Venezuela, posteriormente citada para a Malásia (Marvanová \& Marvan 1969), África (Alasoadura 1968, Ingold 1975), Havaí (Ranzoni 1979), Índia (Sridhar \& Kaveriappa 1989, Rajashekhar \& Kaveriappa 2003). De acordo com Ingold (1975), os representantes desse grupo caracterizam-se por produzir taloconídios terminais, possuidores de um pequeno eixo principal de onde são projetadas ramificações laterais.

Pyramidospora robusta sp. nov. é morfologicamente semelhante a $P$. constricta, espécie descrita por Singh (1972), em associação com folhas submersas no rio Nicol em Sierra Leone, África, e a $P$. densa Alasoadura encontrada em associação a folhas submersas no rio Isasa na Nigéria. Pyramidospora constricta difere dessas por possuir conídios menores e mais delicados $(40-70 \times 1-3 \mu \mathrm{m})$. Essas estruturas produzem um conídio terminal constituído de um eixo principal $(23-47 \times 2-5 \mu \mathrm{m})$ de onde partem duas a cinco ramificações laterais $(8-12 \times 2-5 \mu \mathrm{m})$, sendo que o conídio maduro mede $23-47 \mu \mathrm{m}$. Na espécie $P$. densa os conidióforos são hialinos, muito delicados, simples, eretos, septados transversamente, porém mais longos $(20-50 \times 1,5-3 \mu \mathrm{m})$, produzem também um conídio terminal, hialino, septado possuidor de um eixo 


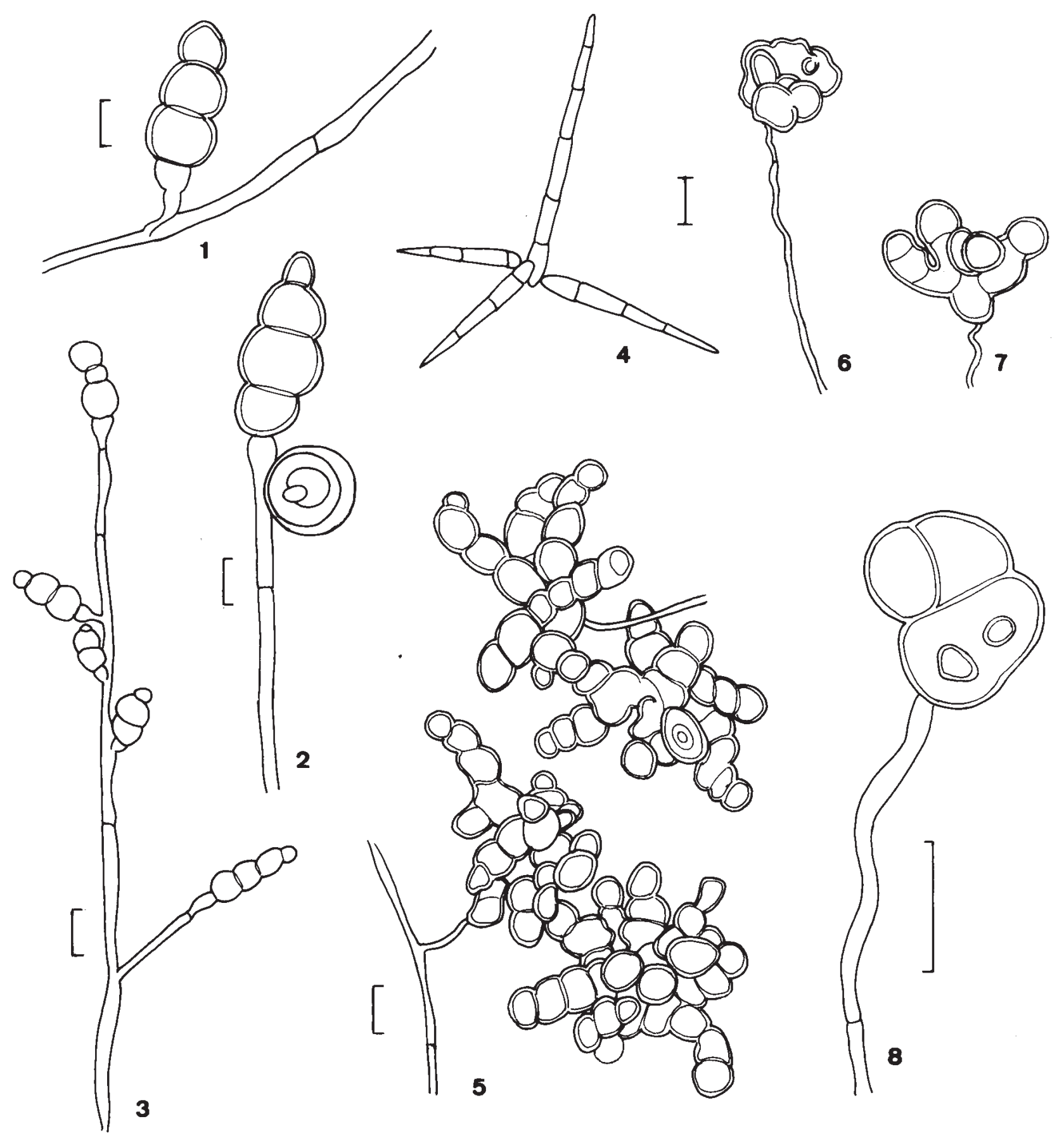

Figuras 1-8. Espécies de hifomicetos associadas ao folhedo de Caesalpinia echinata Lam. e Campomanesia phaea (O. Berg.) Landrum. 1-3. Blodgettia indica. 1. Aspecto geral dos conidioforos com os conídios. 2-3. Conídios. 4. Triscelophorus acuminatus, conídios. 5-8. Pyramidospora robusta sp. nov., conidióforos com conídios maduros. 6-8. Conídios em início de desenvolvimento. Barras de escala $=10 \mu \mathrm{m}(1,2,4-8), 20 \mu \mathrm{m}(3)$.

Figures 1-8. Hyphomycetes species associated with leaf litter of Caesalpinia echinata Lam. and Campomanesia phaea (O. Berg.) Landrum. 1-3. Blodgettia indica. 1. General appearance of conidiophores with conidia. 2-3. Conidia. 4. Triscelophorus acuminatus, conidia. 5-8. Pyramidospora robusta sp. nov., conidiophores with mature conidia. 6-8. Conidia in early development. Scale bars $=10 \mu \mathrm{m}$ $(1,2,4-8), 20 \mu \mathrm{m}(3)$. 

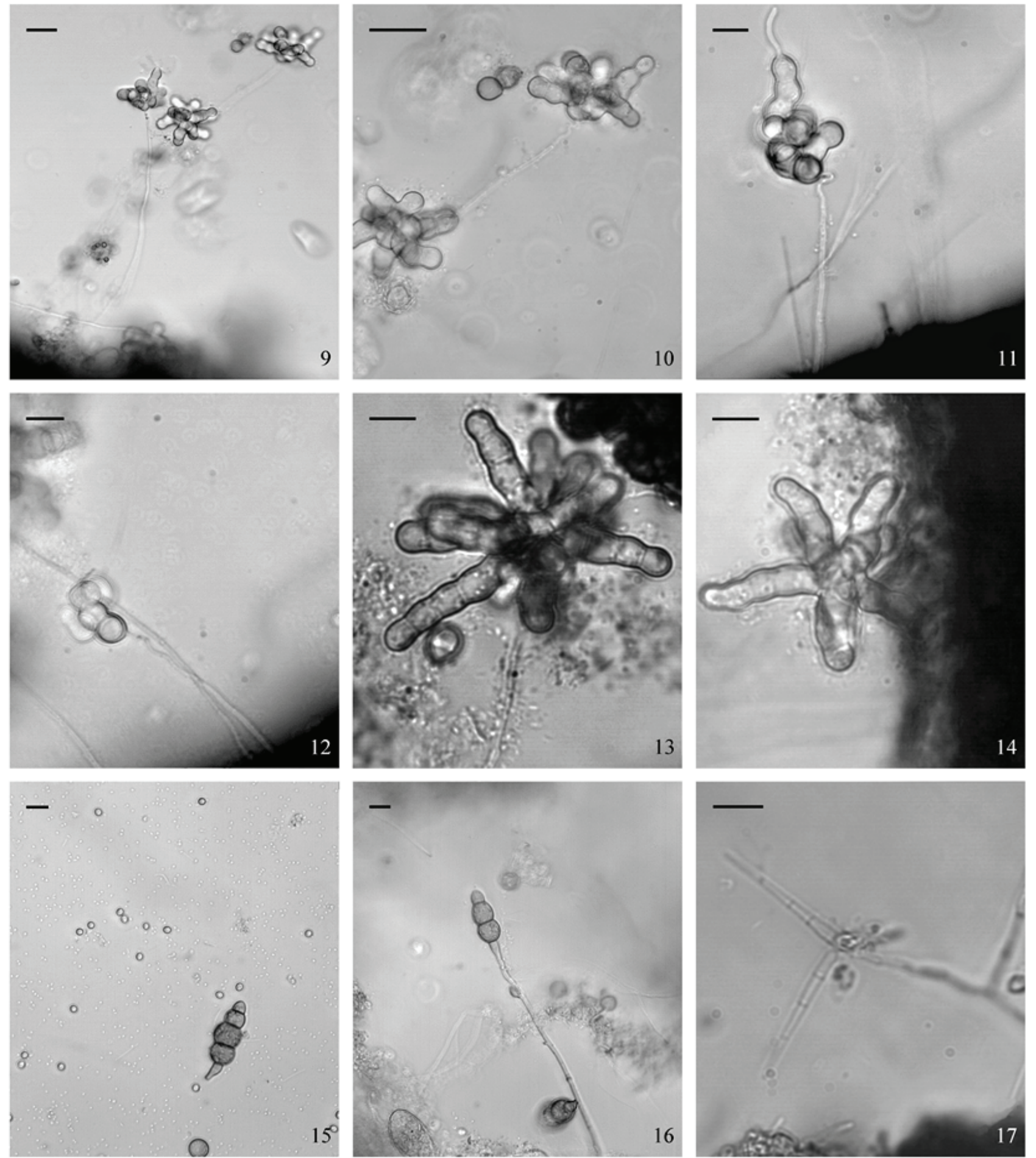

Figuras 9-17. Espécies de hifomicetos associados ao folhedo de Caesalpinia echinata Lam. e Campomanesia phaea (O. Berg.) Landrum. 9-14. Pyramidospora robusta sp. nov. Visão geral dos conidióforos com conídios terminais. 11-12. Início do desenvolvimento do conídio. 13-14. Conídios maduros. 15-16. Blodgettia indica. 15. Conídio. 16. Conidióforo com conídio. 17. Triscelophorus acuminatus, conidióforo com conídio. Barras de escala $=20 \mu \mathrm{m}(9-10), 10 \mu \mathrm{m}$ (11-17).

Figures 9-17. Hyphomycetes species in leaf litter of Caesalpinia echinata Lam. and Campomanesia phaea (O. Berg.) Landrum. 9-14. Pyramidospora robusta sp. nov., overview of conidiophores with terminal conidia. 11-12. Early development of conidia. 13-14. Mature conidia. 15-16. Blodgettia indica. 15. Conidia. 16. Conidiophores with conidia. 17. Triscelophorus acuminatus, conidiophores with conidia. Scale bars $=20 \mu \mathrm{m}(9-10), 10 \mu \mathrm{m}(11-17)$. 
principal $(10-15 \times 4-7,5 \mu \mathrm{m})$ dividido em duas células de onde se projetam 6-8 ramificações $(7-15 \times 4-7,5 \mu \mathrm{m})$, com conídios maduros que medem de 29-37 $\mu \mathrm{m}$.

A espécie proposta no presente estudo possui conidióforos menores e mais delicados. Os conídios são maiores do que os descritos para as duas espécies brevemente discutidas acima e apresentam mais ramificações laterais.

Tipo: BRASIL. São Paulo: São Paulo, Parque Municipal Alfredo Volpi, 25-V-2008, C.G. Moreira \& I.H. Schoenlein-Crusius s.n. (SP416391).

Habitat: folhas de Caesalpinia echinata Lam. e Campomanesia phaea (O. Berg.) Landrum submersas em um lago no Parque Municipal Alfredo Volpi, São Paulo, SP, Brasil.

Triscelophorus acuminatus Nawawi, Trans. Br. Mycol. Soc. 64: 346. 1975.

Figuras 4 e 17

Conidióforos hialinos, simples, eretos, septados transversamente, pouco diferenciados das hifas somáticas e imersos às margens das folhas. Taloconídios são hialinos, terminais, alguns apresentam coloração levemente esverdeada, septados transversal e irregularmente, tetrarradiados com três ramificações verticiladas próximas à base do conídio. Eixo principal 32-63 $\times 2-4 \mu \mathrm{m}$ com 4-7 septações transversais e as ramificações 18-48 $\times$ 2-3 $\mu \mathrm{m}$ com até três septações transversais e base constricta.

Triscelophorus foi descrito por Ingold (1943) e possui T. monosporus Ingold. como espécie tipo. Esse gênero é caracterizado por produzir taloconídios terminais e tetrarradiados, formados por um eixo principal alinhado ao conidióforo e um vértice basal de onde se projetam 3-5 ramificações laterais, cada uma conectada por um istmo estreito ao eixo principal do conídio. As características observadas corroboram a descrição original da espécie T. acuminatus publicada por Nawawi (1975), que a verificou e isolou de folhas em decomposição submersas no rio Gombak na Malásia. De acordo com o autor, a diferença entre T. acuminatus e T. monosporus é pequena, e baseia-se na forma truncada da célula basal e na presença de septos transversais regulares nos conídios de T. acuminatus.

Durante algum tempo foi considerada tropical, mas atualmente a sua distribuição é considerada mais ampla, pois foi encontrada, entre diversas citações, por Matsushima (1981) em associação a folhas submersas em rio da bacia hidrográfica "Saugahatchee Creek" no Alabama, Estados Unidos, por Sati \& Ayra
(2009) no Himalaia e por Betancourt \& Garcia (1987) na Venezuela.

Material examinado: BRASIL. São PAULO: São Paulo, Parque Municipal Alfredo Volpi, 25-V-2008, C.G. Moreira \& I.H. Schoenlein-Crusius s.n. (SP416393).

Habitat: folhas de Caesalpinia echinata Lam. e Campomanesia phaea (O. Berg.) Landrum submersas em um lago no Parque Municipal Alfredo Volpi, São Paulo, SP, Brasil.

\section{Agradecimentos}

Os autores agradecem aos pesquisadores Dr. José Ivanildo de Souza e Dr. Michel Navarro Benatti, pelo auxílio na confecção das ilustrações; à Profa. Dra. Ludmila Marvanová, pela revisão da descrição taxonômica de Pyramidospora robusta; ao Prof. Dr. Tarciso Filgueiras, pela versão para o Latim da descrição da nova espécie; à CAPES, pela bolsa de doutorado concedida à primeira autora; e ao $\mathrm{CNPq}$, pela concessão de bolsa de produtividade à segunda autora (processo 306923/2006-8).

\section{Literatura citada}

Alasoadura, S.O. 1968. Some aquatic Hyphomycetes from Nigeria. Transaction of the British Mycological Society 51: 535-540.

Alexopoulos, C.J., Mims, C.W. \& Blackwell, M. 1996. Introductory Mycology. 4. ed. John Wiley Sons, Inc., New York.

Au, D.W.T., Hodkiss, I.J. \& Vrijmoed, L.L.P. 1992. Fungi and cellulolytic activity with decomposition of Bauhinia purpurea leaf litter in a polluted and unpolluted Hong Kong waterway. Canadian Journal of Botany 70: 1071-1079.

Betancourt, C. \& Garcia, J.C.J. 1987. Los hifomicetos acuaticos De la Quebrada Dona Juana en el Bosque Estatal de Toro Negro, Villalba, Puerto Rico. Caribbean Journal of Science 23: 278-284.

Grandi, R.A.P. \& Gusmão, L.F.P. 1998. A técnica de lavagem sucessiva de substratos de plantas como subsídio para estudos da associação fungo/substrato e diversidade de Hyphomycetes no ecossistemas. In: S. Watanabe (ed.). Anais do IV Simpósio de Ecossistemas Brasileiros, São Paulo, v.3, pp. 80-90.

Gulis, V., Marvanová, L. \& Descals, E. 2005. An illustrated key to the Common temperate species of aquatic Hyphomycetes. In: M.A.S. Graça, F. Bärlocher \& M.O. Gessner (eds.). Methods to study litter decomposition: A Practical Guide. Springer Verlag, Netherlands, pp. 153-167. 
Ingold, C.T. 1943. Triscelophorus monosporus N.Gen., N.SP., an aquatic hyphomycete. Transactions of the British Mycological Society 26: 148-152.

Ingold, C.T. 1975. Guide to aquatic and water-borne Hyphomycetes (Fungi Imperfecti) with notes on their biology. Freshwater Biological Association, Ambleside.

Malosso, E. 1999. Hyphomycetes em ambientes aquáticos lótico e lêntico - ocorrência e biomassa. Dissertação de Mestrado, Universidade Federal de São Carlos, São Carlos.

Marvanová, L. 1997. Freshwater Hyphomycetes: a survey with remarks on tropical taxa. In: K.K. Janardhanan, C. Rajendran, K. Natarajan \& D.L. Hawksworth (eds.). Tropical Mycology. Science Publishers Inc., EUA, pp. 169-226.

Marvanová, M. \& Marvan, P. 1969. Aquatic Hyphomycetes in Cuba. Ceska Mycologie 23: 135-140.

Matsushima, T. 1981. Matsushima Mycological Memoirs n.2. Published by the author. Kobe.

Matsushima, T. 1993. Matsushima Mycological Memoirs n.7. Published by the author. Kobe.

Moreira, C.G. 2006. Avaliação da diversidade e biomassa de fungos associados a folhas em decomposição de Tibouchina pulchra Cogn. submersas em reservatórios do Parque Estadual das Fontes do Ipiranga (PEFI), São Paulo, SP. Dissertação de Mestrado, Instituto de Botânica, São Paulo.

Moreira, C.G. 2011. Sucessão de hifomicetos e avaliação da biomassa fúngica durante a decomposição de folhedo de Caesalpinia echinata Lam. e Campomanesia phaea (O. Berg.) Landrum submersos em lagos artificiais na cidade de São Paulo, SP. Tese de Doutorado, Instituto de Botânica, São Paulo.

Morton, J.B., Bentivenga, S.P. \& Wheeler, W.W. 1993. Germplasm in the International Collection of Arbuscular and Vesicular-arbuscular Mycorrhizal Fungi (INVAM) and procedures for culture development, documentation and storage. Mycotaxon 48: 491-528.

Nawawi, A. 1975. Triscelophorus acuminatus sp. nov. Transactions of the British Mycological Society 64: 345-348.

Nilsson, S. 1962. Some aquatic Hyphomycetes from South America. Svensk Botanisk Tidskrift 56: 351-361.

Pugh, G.J.F., Buckey, N.G. \& Mulder, J. 1972. The role of phylloplane fungi in the early colonization of leaves. Symposia Biológica Hungarica 11: 329-333.
Rajashekar, M. \& Kaveriappa, K.M. 2003. Diversity of aquatic Hyphomycetes in the aquatic ecosystem of the Western Ghats of India. Hydrobiologia 501: 167-177.

Ranzoni, R.V. 1979. The Aquatic Hyphomycetes from Hawaii. Mycologia 71: 786-795.

Sati, S.C. \& Arya, P. 2009. Occurrence of aquatic water borne conidial fungi in relation to some physical-chemical parameters in a freshwater stream. Nature and Science 7: 20-28.

Schoenlein-Crusius, I.H. \& Grandi, R.A.P. 2003. The diversity of Aquatic Hyphomycetes in South America. Brazilian Journal of Microbiology 34: 183-193.

Schoenlein-Crusius, I.H. \& Malosso, E. 2007. Diversity of aquatic Hyphomycetes in the tropics. In: B.N. Ganguli \& S.K. Deshmukh (eds.). Fungi: Multifaceted microbes. Anamaya Publishers, New Delhi, pp. 61-81.

Schoenlein-Crusius, I.H. 2002. Aquatic Hyphomycetes from cerrado regions in the state of São Paulo, Brazil. Mycotaxon 81: 457-462.

Schoenlein-Crusius, I.H., Milanez, A.I., Trufem, S.F.B. \& Pires-Zottarelli, C.L.A. 2009. Fungos: estudos ecológicos (Capítulo 13). In: M.I.M.S. Lopes, M. Kirizawa \& M.M.R.F. Melo (eds.). Patrimônio da Reserva Biológica do Alto da Serra de Paranapiacaba: a antiga Estação Biológica do Alto da Serra. Instituto de Botânica, São Paulo, pp. 229-241.

Schoenlein-Crusius, I.H., Moreira, C.G. \& Bicudo, D.C. 2009. Aquatic Hyphomycetes in the Parque Estadual das Fontes do Ipiranga-PEFI, São Paulo, Brazil. Revista Brasileira de Botânica 32: 411-426.

Singh, N. 1972. Pyramidospora constricta sp.nov., a new aquatic Hyphomycetes. Transactions of the British Mycological Society 59: 336-340.

Sridhar, K.R. \& Kaveriappa, K.M. 1989. Observations on aquatic Hyphomycetes of the western Ghat streams, India. Nova Hedwigia 49: 455-467.

Subramanian, C.V. 1954. Fungi imperfecti from Madras - VI. Journal of the Indian Botanical Society 33: $36-42$.

SVMA (Secretaria do Verde e do Meio Ambiente da Prefeitura do Município de São Paulo). 2006. Relatório preliminar do estado dos lagos dos parques municipais de São Paulo. http://www.prefeitura.sp.gov// arquivos/secretaria/meio_ambiente/publicacao/lagos publicacao_final.pdf (acesso em 05.10.2007). 
